\title{
Influence of the ambipolar-to-free diffusion transition on dust particle charge in a complex plasma afterglow
}

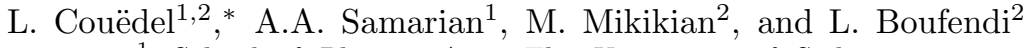 \\ 1 School of Physics A28, The University of Sydney, \\ NSW 2006, AUSTRALIA \\ ${ }^{2}$ GREMI (Groupe de Recherches sur l'Énergétique des Milieux Ionisés), \\ CNRS/Université d'Orléans, 14 rue d'Issoudun, \\ 45067 Orléans Cedex 2, FRANCE
}

(Dated: July 16, 2008)

\begin{abstract}
The influence of diffusive losses on residual dust charge in a complex plasma afterglow has been investigated. The residual charge distribution was measured and exhibits a mean value $Q_{d_{\text {res }}} \sim$ $(-3 e-5 e)$ with a tail in the positive region. The experimental results have been compared with simulated charge distributions. The dust residual charges were simulated based on a model developed to describe complex plasma decay. The experimental and simulated data show that the transition from ambipolar to free diffusion in the decaying plasma plays a significant role in determining the residual dust particle charges. The presence of positively charged dust particles is explained by a broadening of the charge distribution function in the afterglow plasma.
\end{abstract}

PACS numbers: 52.27.Lw

Keywords: Discharging, dust, plasma, residual charges, afterglow

\section{INTRODUCTION}

Dusty plasmas (also called complex plasmas) are ionized gases containing dust particles. These dust particles are electrically charged due to interaction with ions and electrons of the surrounding medium. In laboratory experiments, these particles can be either injected or grown directly in the plasma. Injected dust particles are usually micron-size particles. Due to their mass, they are confined in the sheath region where the electric force counterbalances gravity. Microgravity conditions are thus necessary to study dust clouds of micrometer size particles filling the whole plasma chamber [1]. In the laboratory, dense clouds of sub-micron particles light enough to completely fill the gap between the electrodes can be obtained using reactive gases such as silane $[2,3]$ or using a target sputtered with ions from the plasma [4-9]. The dust particles are also subject to other forces in the plasma such as the ion drag force, the neutral drag force and the thermophoretic force $[10,11]$.

Dust particle charge is a key parameter in a complex plasma. It determines the interaction between a dust particle and electrons, ions, its neighboring dust particles, and electric field. [10,11]. Data of the dust charge will allow us to understand the particle dynamics in dust clouds, and methods of manipulating the particles. The knowledge of the dust charge is very important in every dusty medium (complex plasmas, colloidal suspensions, fog). For example, in gas flow containing dust particles the dust charge and dust charge distribution are studied because of their consequences (safety hazard, dry coat-

${ }^{*}$ Electronic address: lenaic.couedel@univ-orleans.fr ing, etc) [12, 13]; the charging of fog particles is also studied as the electrical properties of fog are very important for the fog forecast and control; and aerosol and dust systems influence the electric fields and currents in the atmosphere [14].

There are many publications reporting on the investigation of dust charging in a discharge plasma; see $[10,15-$ $22]$ and references therein. However there are only a few papers devoted to dust charging, or discharging to be more specific, in the discharge afterglow [23-25]. These papers report that dust particles retain residual electric charges when the power of the discharge is switched off. Nevertheless, the discharging phenomenon was not totally understood. The models proposed do not predict accurately residual charge values observed in those experiments, nor do they explain the existence of positively charge particles.

One of the main reasons is that the models are working with simple assumptions. For example it was assumed [24] that ambipolar diffusion continues until the ionic Debye length $\lambda_{D i}$ reaches the diffusion length $\Lambda$, and then an abrupt transition to free diffusion of ions and electrons occurs. In contrast, it has been shown that diffusion of charged species in a plasma afterglow deviates from ambipolar diffusion as soon as the ratio $\left(\Lambda / \lambda_{D e}\right) \sim 100$ where $\lambda_{D e}$ is the electron Debye length [26-29]. These results indicate that electrons and ions are lost at different rates very early in the decay process and one has to expect that this will strongly affect the value of residual dust charge. As the charge on dust particles is related to the ratio $n_{i} / n_{e}$ where $n_{i(e)}$ is the ion (electron) density [10], this must result in a different dust particle charge evolution in the afterglow compared to the predictions based on previous assumptions [24, 25]. For this reason, a model taking into account the actual transition from 
ambipolar to free diffusion of electrons and ions is vital for the correct calculation of dust charges in afterglow plasma.

In this paper we report numerical simulations of residual electric charge on dust particles in an afterglow plasma for various transitions from ambipolar to free diffusion. These numerical results are compared to the experimental data. The importance of the transition from ambipolar to free diffusion in the decaying plasma for determining the dust particle residual charges is shown. The broadening of the charge distribution function is found to be responsible for the presence of positively charged particles.

\section{MODELING OF DISCHARGE AFTERGLOW}

In this section, we first describe a qualitative model which helps us to explain the existence of residual charges. Then we consider dust-free plasma decay taking into account the experimental transition from ambipolar to free diffusion. Then we discuss the plasma decay in the presence of dust particles using a model for particle charging (discharging) in a afterglow plasma.

\section{A. Four stage model}

The evolution of dust charge in afterglow plasma will depend on the evolution of plasma parameters. In a decaying plasma (afterglow plasma), the kinetics of plasma losses is mainly governed by the electron temperature relaxation process and plasma diffusion and recombination processes [30]. The proposed model in Ref.[25] predicts evolution of dust residual charge after the discharge is switched off. Based on the plasma decay kinetics we can define four stages in a discharge afterglow during which the different processes (electron temperature relaxation, ambipolar diffusion, free diffusion) govern the evolution of dust charge:

1. Electron temperature relaxation stage: the electron temperature decreases to the gas temperature while the plasma density remains almost constant resulting in a strong decrease of dust particle charges ( $t \sim 500 \mu \mathrm{s}$ for an argon pressure $\left.P_{A r} \sim 1.2 \mathrm{mbar}\right)$.

2. Ambipolar diffusion stage: the dust particle charge stays almost constant while plasma particles (ions and electrons) are lost by ambipolar diffusion on to the walls of the reactor. The ambipolar diffusion of ions and electrons continues until the Debye length $\lambda_{D}$ equals the diffusion length $\Lambda$ of the reactor or until the Havnes parameter $P_{H}=\left|\bar{Q}_{d} n_{d} / e n_{e}\right| \sim 1($ $t \sim 40 \mathrm{~ms}$ for an argon pressure $\left.P_{A r} \sim 1.2 \mathrm{mbar}\right)$

3. Free diffusion stage: this stage begins when diffusion changes from ambipolar to free, i.e. electrons and ions start diffusing independently. As electrons diffuse faster than ions, the ratio $n_{i} / n_{e}$ becomes more than one resulting in a decrease of the dust particle charge( $t \sim 60 \mathrm{~ms}$ for an argon pressure $P_{A r} \sim 1.2$ mbar $)$.

4. Finally, ion and electron densities become too small to influence dust particle charges which can be considered as frozen (i.e. charging time tends to infinity).

This model predicts the existence of a negative residual charge of a few electrons but is unable to predict a positive residual charge. The limits of the model are the step transition from ambipolar to free diffusion and the failure to properly take account of the influence of dust particles. Indeed the effect of the dust particles is ignored until the dust charge density is the same as the electron density, which leads to an abrupt change from ambipolar to free diffusion of ions and electrons. The assumption of the step transition from ambipolar to free diffusion is quite unphysical and contradicts existing experimental data [26-29]. These data indicate that electrons and ions are diffusing at different rates very early in the decay process. This smooth transition must lead to a change in dust charges as the ratio $n_{i} / n_{e}$ will be modified early in the decaying process. Under the conditions of our experiments, the ratio $\left(\Lambda / \lambda_{D e}\right) \sim 50$ when the discharge is switched off. Consequently, this must result in a different dust particle charge evolution in the afterglow compared to previous assumptions $[24,25]$. For this reason, in the next sections, a modified model taking into account the gradual transition from ambipolar to free diffusion is used to simulation of complex plasma afterglow.

\section{B. Dust free plasma decay}

In a dust-free plasma of monoatomic gas, losses of the plasma are mainly due to diffusion to the walls. At the very beginning of the afterglow when the discharge is switched off, plasma density $n$ follows:

$$
\frac{d \tilde{n}}{d t}=-\frac{\tilde{n}}{\tau_{D}}
$$

where $\tilde{n}=n / n_{0}$ with $n_{0}$ the initial plasma density and $\tau_{D}$ is the time scale for ambipolar diffusion given by [30]:

$$
\tau_{D}^{-1}=\frac{D_{a}}{\Lambda^{2}} \simeq \frac{l_{i n} v_{T_{i}}}{3 \Lambda^{2}}\left(1+\tilde{T}_{e}\right) \equiv \frac{1}{2}\left(1+\tilde{T}_{e}\right) \frac{1}{\tau_{D}^{\infty}}
$$

where $D_{a}$ is the ambipolar diffusion coefficient, $\tilde{T}_{e}=$ $T_{e} / T_{i}$ with $T_{e}$ the temperature of electrons, $T_{i}=T$ the temperature of ions and $T$ the temperature of neutral atoms, $v_{T_{i}}=\sqrt{8 k_{B} T / \pi m_{i}}$ the thermal speed of ions with $k_{B}$ the Boltzmann constant and $m_{i}$ the ion mass 
and $l_{i n}=1 /\left(n_{n} \sigma_{i n}\right)$ the mean free path for ion-neutral collisions, $n_{n}$ the density of neutral atom and $\sigma_{i n}$ the ion-neutral cross section.

Nevertheless, it has been shown that the plasma diffusion deviates from ambipolar diffusion as soon as the ratio $\left(\Lambda / \lambda_{D e}\right) \sim 100$ [26-29]. From this moment, ions and electrons must be treated separately. In our model, the deviation from ambipolar diffusion is derived from the results of Gerber and Gerardo [29] or Freiberg and Weaver [26]. Both experiments performed in helium at different pressures exhibit similar evolution of the diffusion coefficients. Though these results where obtained with helium, they can be used to estimate the transition in an argon plasma. Indeed, the ambipolar diffusion coefficient is:

$$
D_{a} \propto \frac{\left(k_{B} T_{i}\right)^{3 / 2}}{P \sigma_{i n} m_{i}^{1 / 2}}
$$

where $P$ is the neutral gas pressure. As $\sigma_{i n_{A r+}} \sim$ $2.5 \sigma_{i n_{H^{+}}+}[31]$ and $m_{i_{A r}+} \sim 10 m_{i_{H e}+}, D_{a_{A r}} \sim(1 / 8)$. $D_{a_{H e}}$ for equal pressures. Consequently, it can be assumed that the diffusion of an argon plasma with argon pressure $P_{A r}$ is the same as a helium plasma with helium pressure $P_{H e} \simeq 8 \cdot P_{A r}$. The experimental results obtained for Helium are in the range of pressure $0.4-4$ Torr [26] or $9-22.8$ Torr [29] which correspond to $0.05-0.5$ Torr and $1.13-2.85$ Torr respectively in equivalent argon pressure. Consequently, these sets of data can be used for upper and lower estimation in the simulations which are performed in the range $0.3-1$ Torr $(0.4-1.3$ mbar $)$.

In the model, electron and ion densities are treated separately and follow:

$$
\begin{aligned}
\frac{d n_{i}}{d t} & =-\frac{n_{i}}{\tau_{D i}} \\
\frac{d n_{e}}{d t} & =-\frac{n_{e}}{\tau_{D e}}
\end{aligned}
$$

where $n_{i(e)}$ is the ion (electron) density and $\tau_{D i(e)}$ is the ion (electron) diffusion time. The diffusion times are function of the ratio $\left(\Lambda / \lambda_{D e}\right)^{2}$ and are calculated using experimental results from Gerber and Gerardo [29] or Freiberg and Weaver [26] (see Fig.1) (only results from Ref.[29] are available for ions and, consequently, there have been used).

The electron temperature relaxes due to energy exchange in collisions with neutrals. At the initial stage of plasma decay the electron temperature $T_{e 0}$ is much higher than the neutral temperature $T$ and will decrease and tend asymptotically to $T$. The equation for electron temperature relaxation is [30]:

$$
\frac{d \tilde{T}_{e}}{d t}=-\frac{\tilde{T}_{e}-1}{\tau_{T}}
$$

where $\tau_{T}^{-1}=\sqrt{\pi / 2} \sqrt{m_{e} / m_{i}} v_{T_{i}} / l_{e n} \sqrt{\tilde{T}_{e}} \equiv \sqrt{T_{e}} / \tau_{T}^{\infty}, m_{e}$ is the electron mass and $l_{e n}$ is the mean free path of

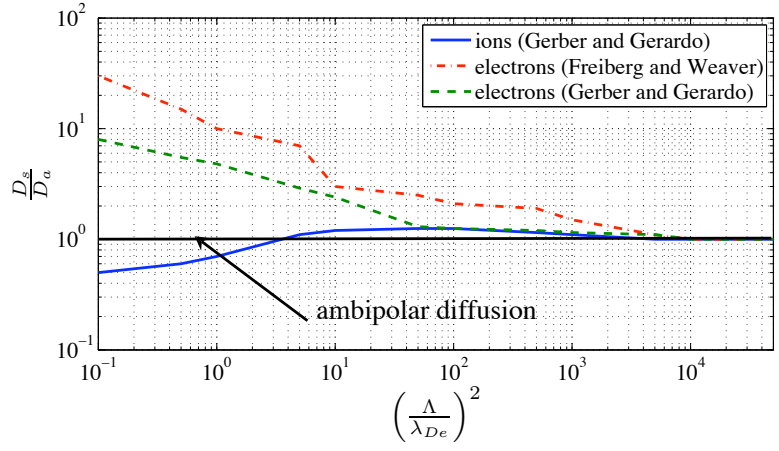

FIG. 1: (Color online) Evolution of the ratio $D_{s} / D_{a}(s=i, e)$ as a function of $\left(\Lambda / \lambda_{D e}\right)^{2}$. These data are extracted from Refs.[29] and [26].

electron-neutral collision. It can be deduced from Eq.6 that the electron cooling time decreases when increasing the pressure.

\section{Plasma decay in the presence of dust}

In the presence of dust particles in the plasma, the temperature relaxation and diffusion losses could be different from dust-free plasma. However, no experimental data nor theoretical estimations of the transition from ambipolar to free diffusion in dusty plasma exist. Consequently, for our simulation we will assume that the electron temperature relaxation and diffusion processes of ions and electrons are not significantly affected by the presence of dust particles, allowing us to treat ion and electron diffusion in a similar way to dust-free plasma. This restricts the validity of our simulations to the case of low dust particle densities. In this case, the influence of dust particles can be restricted to plasma absorption losses on the particle surface. Indeed, when immersed in a plasma, a dust particle acquires a net electric charge due to ions and electrons "falling" on its surface $[10,11,32]$. In dusty plasmas, ions and electrons captured by dust particles can be considered as "lost" by the plasma and thus the charging process of dust particles is also a loss process. In the Orbital Motion Limited (OML) approach [10, 11, 32 , the charge on a dust particle can be calculated from the ion and electron currents ignoring emission currents:

$$
\frac{d Q_{d}}{d t}=I_{e}+I_{i}
$$

where $Q_{d}$ is the charge on the dust particle and $I_{e(i)}$ is the electron (ion) current. In a typical laboratory discharge, dust particles are negatively charged due to higher mobility of the plasma electrons. The ion current can be expressed as:

$$
I_{i}=4 \pi r_{d}^{2} n_{i} q_{i}\left(\frac{k_{B} T_{i}}{2 \pi m_{i}}\right)^{1 / 2}\left(1-\frac{q_{i} \phi_{d}}{k_{B} T_{i}}\right)
$$


and the electron current as:

$$
I_{e}=4 \pi r_{d}^{2} n_{e} q_{e}\left(\frac{k_{B} T_{e}}{2 \pi m_{e}}\right)^{1 / 2} \exp \left(-\frac{q_{e} \phi_{d}}{k_{B} T_{e}}\right)
$$

where $\phi_{d}$ is the surface potential of the dust particle, $r_{d}$ is the dust particle radius and $q_{i(e)}$ is the ion (electron) charge. In steady state, the charge on a dust particle can be obtained:

$$
Q_{d}=C \cdot \frac{4 \pi \epsilon_{0} r_{d} k_{B} T_{e}}{e} \ln \left(\frac{n i}{n_{e}}\left(\frac{m_{e} T_{e}}{m_{i} T_{i}}\right)^{1 / 2}\right)
$$

For typical argon plasma $m_{e} / m_{i} \approx 1.4 \cdot 10^{-5}$ and $1<$ $T_{e} / T_{i}<100$ and the correction factor $C \sim 0.73$ [33].

A dusty afterglow plasma is not a steady case but the OML approach can be used to obtain the charge on dust particles. A Fokker-Planck model of dust charging due to the discreetness of the charge currents (ion and electron) can be used to obtain dust particle charge distributions. Indeed, the plasma particle absorption time interval as well as the sequence in which electrons and ions arrive at the dust particle surface vary randomly but observe probabilities that depend on the dust particle potential $\phi_{d}$. The probability per unit of time for absorbing an electron $p_{e}\left(\phi_{d}\right)$ or an ion $p_{i}\left(\phi_{d}\right)$ are calculated from the OML currents [34]:

$$
\begin{aligned}
p_{e} & =-I_{e} / e \\
p_{i} & =I_{i} / q_{i}
\end{aligned}
$$

The ion and electron currents can be calculated using Eq. 8 and Eq.9 only when the dust particle charge is negative. For positively charged dust particles, the ion current is calculated by Eq.9 with all subscripts changed to $i$ and electron current is calculated by Eq. 8 with all subscripts changed to $e$. In the simulation, electron and ion densities are studied in a fluid manner solving numerically Eqs. 4 and 5 using the same constant time step $t_{f}<<\tau_{D i(e) 0}$ where $\tau_{D i(e) 0}$ is the diffusion time for ions (electrons) at the beginning of the simulation (i.e the very beginning of the decaying plasma).

The contribution of dust particles to plasma losses is taken into account in the following way:

The charges of $N_{d}$ dust particles are computed. Knowing the dust density $n_{d}$ in the plasma, an equivalent volume $V_{e q}$ to these $N_{d}$ dust particles can be calculated.

For a dust particle, a time step $t_{p_{j}}$ for which the probability of collecting an ion or an electron of the plasma is computed [34]:

$$
t_{p_{j}}=-\frac{\ln (1-R 1)}{p_{t o t}}
$$

where $0<R 1<1$ is a random number and $p_{t o t}=p_{e}+p_{i}$ is the total probability per unit of time to absorb an ion or an electron.

While $\sum_{j} t_{p_{j}} \leq t_{f}$, an electron or an ion is absorbed during the time step $t_{p_{j}}$ and the nature of the absorbed particle is determined comparing a second random number $0<R 2<1$ to the ratio $p_{e} / p_{t o t}$. If $R 2<p_{e} / p_{t o t}$, then the collected particle is an electron otherwise it is an ion. The probability $p_{e}$ and $p_{i}$ are recalculated and a new time step $t_{p_{j+1}}$ is computed.

When $\sum_{j} t_{p_{j}}>t_{f}$, one more iteration is applied to the dust particle. Nevertheless, the probability of collecting an ion or an electron in the time interval $\Delta t=t_{f}-\sum_{k=1}^{j-1} t_{p_{k}}$ is $p(\Delta t)=1-\exp \left(-\Delta t \cdot p_{t o t}\right)$ (if $t_{p_{1}}>t_{f}$ then $p\left(t_{p_{1}}\right)$ is computed). A random number $0<R 3<1$ is then generated and if $R 3<p(\Delta t)$ a plasma particle (ion or electron) is absorbed. The choice between ion and electron is decided as previously described.

When all the $N_{d}$ dust particles have been treated, the number of absorbed ions and electrons $N_{\text {iabs }}$ and $N_{\text {eabs }}$ respectively by the $N_{d}$ dust particles during the time step $t_{f}$ is known and can be transformed using the equivalent volume $V_{e q}$ into absorbed ion and electron densities $n_{i a b s}$ and $n_{e a b s}$ respectively which are subtracted from the ion density $n_{i}$ and electron density $n_{e}$ before the next iteration of time step $t_{f}$.

For each iteration, the mean time $\overline{t_{p}}$ necessary for one particle to collect a plasma particle (i.e. an ion or an electron) as well as the ratio $r_{l o s s}=n_{e(i)_{d i f f}} / n_{e(i)_{a b s}}$ where $n_{e(i)_{\text {diff }}}$ is the density of electron (ion) lost by diffusion. The program is stopped when $\overline{t_{p}}>>\tau_{D i}$ ( $\overline{t_{p}}>10 \cdot \tau_{D i}$ in this simulation) when the charge on dust particles can be considered as frozen due to a plasma loss time becoming faster than the particle charging time.

\section{RESULTS AND DISCUSSION}

In this section, the experimental dust charge distributions as well as the simulated ones are presented.Then we discuss the validity of our results and role of the transition from ambipolar to free diffusion.

\section{A. Experimental results}

The experimental residual charge distributions have been obtained using the PKE-Nefedov reactor [1]. An upward thermophoretic force was applied to dust particles in order to counterbalance gravity [35] when the discharge is off. To study particle charges, a sinusoidal voltage produced by a function generator with amplitude $\pm 30 \mathrm{~V}$ and frequency of $f=1 \mathrm{~Hz}$ was applied to the bottom electrode. The induced low frequency sinusoidal electric field generates dust oscillations if the dust particles have a residual electric charge. A thin laser sheet perpendicular to the electrodes illuminates the particles and the scattered light is recorded at $90^{\circ}$ with standard charge coupled device (CCD) cameras. By superimposition of video frames, particle trajectories have been ob- 
tained. The coordinates of the particles were measured in each third frame and the amplitude of the oscillations was determined from the measured particle positions. Absolute values for the oscillation amplitude were obtained by scaling the image pixels to the known size of the field of view.

The charge of a dust particle $Q_{d}$ can then be obtained from the oscillation amplitude $b$ [25]:

$$
Q_{d_{r e s}}=\frac{m_{d} b \omega \sqrt{\omega^{2}+4 \gamma^{2} / m_{d}^{2}}}{E_{0}}
$$

where $m_{d}$ is the mass of the dust particle, $E_{0}$ is the amplitude of the electric field at the mean height of the dust particle, $\omega=2 \pi \cdot f$ the angular frequency of the sinusoidal electric field and $\gamma$ the damping coefficient. The electric field $E_{0}$ was taken as the one above the axis of a polarized disk. For a levitation height of $1.5 \mathrm{~cm}$ the electric field is $E_{0}=6 \mathrm{~V} / \mathrm{cm}[25]$. The total error on the residual charge measurement (taking into account the error on the dust mass, dust size, $E_{0}$, and the damping force) is about $\sim 49 \%$. The sign of the dust particle charge is deduced from the phase of the dust particle oscillation with respect to the excitation electric field. A more detailed description of this experiment can be found in Ref.[25].

Following and measuring oscillations of many dust particles, the dust particle residual charge distribution in a afterglow plasma can be constructed. In Fig.2, dust particle residual charge distributions are presented for two operating pressures. It can be seen that for
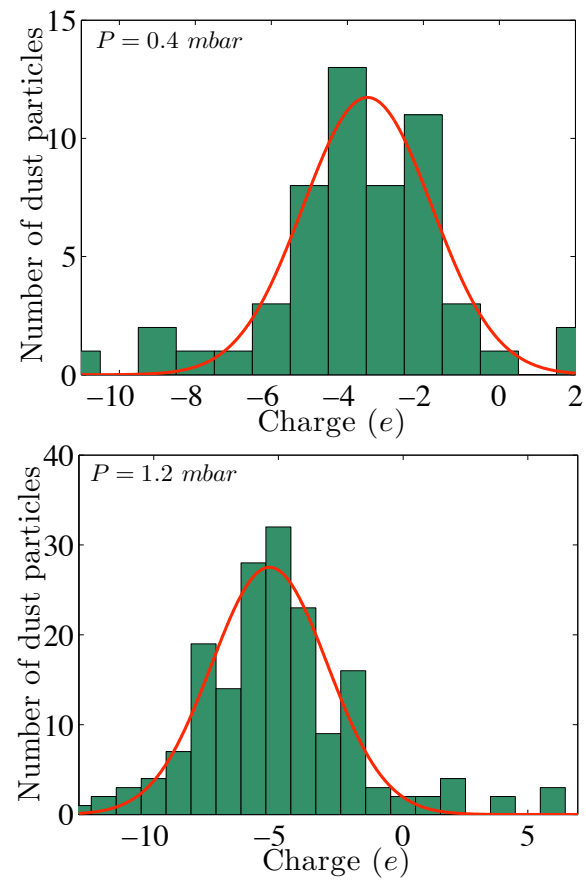

FIG. 2: (Color online) Experimental dust charge distribution. Top: $P=0.4$ mbar. Bottom $P=1.2$ mbar. A gaussian fit is superimposed to the experimental distributions both pressures the mean residual charge is negative and corresponds to a few electrons (-5e for $1.2 \mathrm{mbar}$ and $-3 e$ for 0.4 mbar). In both cases, the measured residual charge distribution has a tail that extends into positive residual charge region.

\section{B. Numerical results}

The numerical dust particle charge distributions are reconstructed simulating the charge of $N_{d}=500$ dust particles corresponding to a dust density $n_{d}=5 \cdot 10^{4} \mathrm{~cm}^{-3}$. The dust particle radius was $r_{d}=190 \mathrm{~nm}$ corresponding to the experimental ones. The initial ion density is $n_{i 0}=5 \cdot 10^{9} \mathrm{~cm}^{-3}$ and the initial dust particle charge distribution is computed using a Cui-Goree algorithm [34] and the quasi-neutrality condition:

$$
Z_{d} n_{d}+n_{e}=n_{i}
$$

where $Z_{d}=\left|Q_{d} / e\right|$. The initial electron density $n_{e 0}$ is deduced from this calculation. Many iterations of the algorithm are necessary to obtain the initial dust charge distribution and the initial electron density: the first iteration assumes $n_{e 0}=n_{i 0}=5 \cdot 10^{9} \mathrm{~cm}^{-3}$, and for the next iteration $n_{e 0}$ is calculated using Eq.15 and the dust particle charges of the first iteration. A new dust particle charge distribution is then computed. This process is performed again and again until $n_{e 0}$ and the dust charge distribution are stabilized. The obtained dust particle charge distribution is presented in Fig. 3 The mean charge

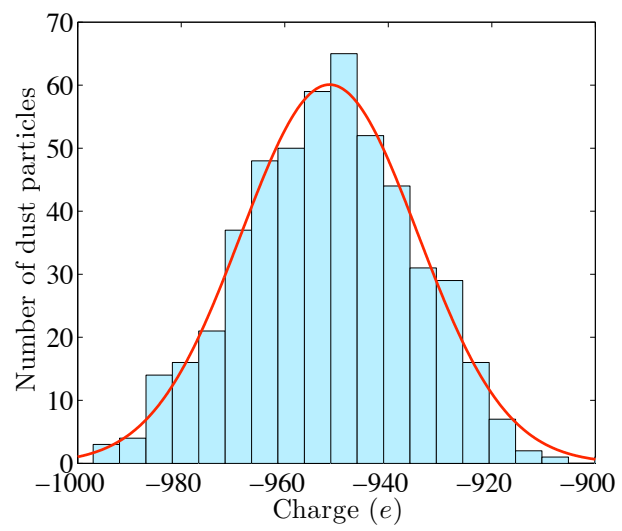

FIG. 3: (Color Online) Dust charge distribution for $190 \mathrm{~nm}$ radius dust particles with $n_{i 0}=5 \cdot 10^{9} \mathrm{~cm}^{-3}$ and $n_{d}=5$. $10^{4} \mathrm{~cm}^{-3}$

is $Q_{\text {mean }} \simeq-952 e$ and the variance $\sigma\left(Q_{d}\right) \simeq 17 e$. Eq.10 predicts dust particle charges $Q_{d}=-950 e$ which is in total agreement with our simulation results.

The decay of a dusty argon plasma is then simulated using the algorithm previously described for two gas pressures $(P=0.4 m b a r$ and $P=1.2 m b a r$ (Fig. 4$))$. The ionneutral mean free path is calculated using the cross sec- 

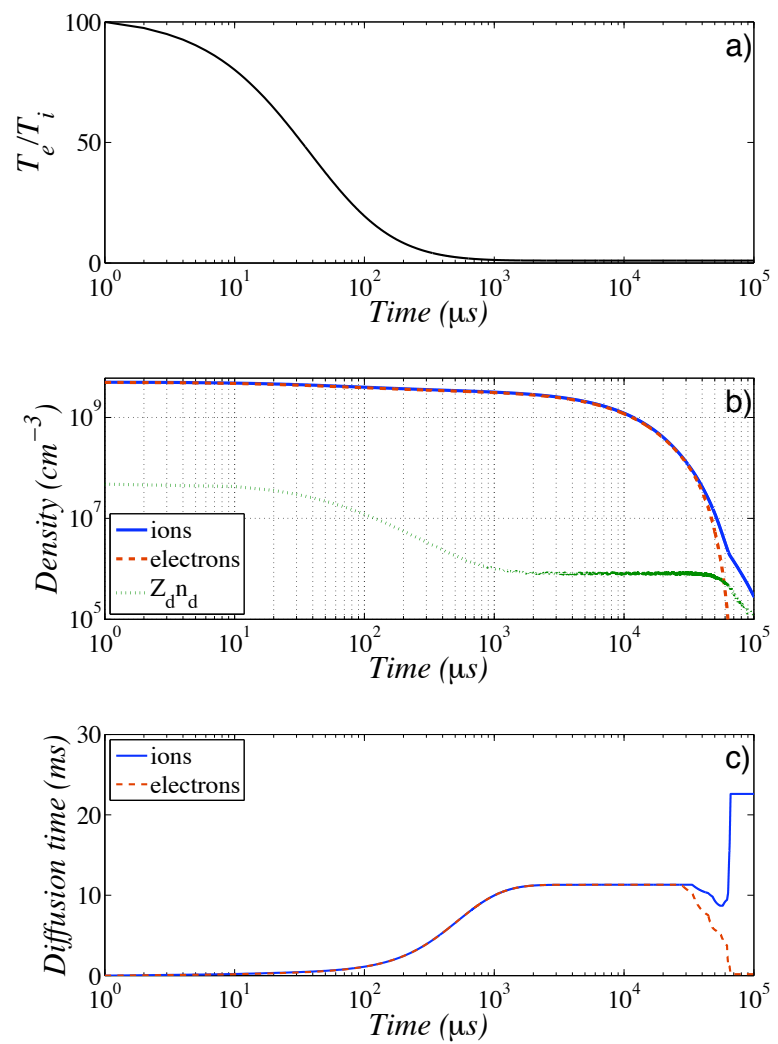

FIG. 4: (Color Online) Decay of an argon plasma at $P=$ 1.2 mbar with a fast ambipolar-to-free diffusion transition. a) Electron temperature relaxation; b)density evolution; c) evolution of diffusion time.

tion from Varney [31] and the electron-neutral mean freepath is calculated using the cross section from Kivel [36]. The initial electron temperature is taken as $T_{e 0}=3 \mathrm{eV}$ and the ion temperature is supposed to be equal to the neutral temperature $T_{i}=T_{n}=T=0.03 \mathrm{eV}$. The diffusion length is taken $\Lambda=1 \mathrm{~cm}$ which is approximately the diffusion length of the PKE-Nefedov reactor in which experiments on residual dust charge have been performed $[24,25]$. The transition from ambipolar to free diffusion is based on either experimental results from Gerber and Gerardo [29] or experimental results from Freiberg and Weaver [26] (the former suggest a slower transition from ambipolar to free diffusion than the latter, see Fig.1).

As it can be seen in Fig.4, the first decrease of the dust particle charge corresponds to the electron temperature relaxation. Then, while electrons and ions diffuse ambipolarly, the charge remains constant. Finally, when the transition occurs (after tens of ms), electron and ions densities deviate from each other and the dust charge deacreases until it freezes.

For a pressure $P=0.4 m b a r(P=0.3$ Torr $)$, the simulated final dust charge distributions (i.e. $\overline{t_{p}}>10 \cdot \tau_{D i}$ ) are presented in fig.5. The residual charge is $Q_{d_{\text {res }}} \simeq-16 e$ when no transition in the diffusion process is taken into account (Fig.5a). When using a model based on an
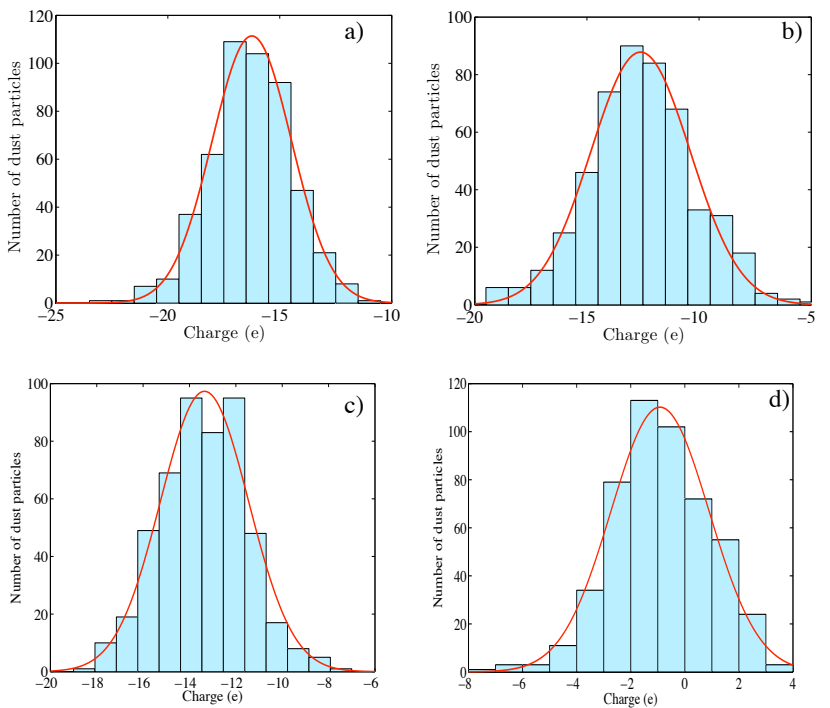

FIG. 5: (Color Online) Numerical results for $190 \mathrm{~nm}$ radius dust particles with argon pressure $P=0.4$ mbar $(P=$ 0.3 Torr $)$ and $n_{d}=5 \cdot 10^{4} \mathrm{~cm}^{-3}$. a) Ambipolar diffusion until the end of the decay process. b) Abrupt transition from ambipolar to free diffusion when $P_{H}=0.5$. c) Using data from Gerber and Gerardo for transition from ambipolar to free diffusion [29]. d) Using data from Freiberg and Weaver for transition from ambipolar to free diffusion [26]

abrupt transition from ambipolar to free diffusion at a Havnes parameter of $P_{H}=0.5$, the residual charge is smaller (in absolute value) $Q_{d_{\text {res }}} \simeq-13 e$ but still far from experimental value (Fig.5b). It should be noted that, typically, the Havnes parameter reaches 0.5, after $10 \mathrm{~ms}$; it corresponds to a $\Lambda / \lambda_{D e}$ ratio close to unity. Using Gerber and Gerardo data (slow transition, lower curve in Fig.1), the mean residual charge $Q_{d_{\text {res }}} \simeq-13 e$ (see Fig.5c) whereas it is $Q_{d_{\text {res }}} \simeq-1 e$ (see Fig.5d) using Freiberg and Weaver data (fast transition, higher curve in Fig.1). In the slow transition case, there are no positive particles observed in the simulated dust particle charge distribution whereas there are ones in the case of the fast transition. The residual charge distribution for the fast transition is similar to the experimental results.(see Fig.2)

For a pressure $P=1.2 \operatorname{mbar}(P=0.9$ Torr $)$, the simulated final dust particle charge distributions are presented in fig.6. The residual charge is $Q_{d_{\text {res }}} \simeq-16 e$ when no transition in the diffusion process is taken into account (Fig.6a). When using a model based on an abrupt transition from ambipolar to free diffusion (at a Havnes parameter of $P_{H}=0.5$ ), the residual charge is smaller (in absolute value) $Q_{d_{\text {res }}} \simeq-7 e$ but still far from experimental value (Fig.6b). A dependence on the ambipolar to free diffusion transition is again seen with $Q_{d_{\text {res }}} \simeq-14 e$ for the slow transition and $Q_{d_{r e s}} \simeq-2 e$ for the fast transition. The latest charge distribution is the closest to experimentally measured distribution. 
TABLE I: Dust particle residual charges for two operating pressures.

\begin{tabular}{ccc}
\hline \hline & $P=1.2$ mbar $P=0.4$ mbar \\
\hline Experimental results & $-5 e$ & $-3 e$ \\
Ambipolar diffusion & $-16 e$ & $-16 e$ \\
Havnes transition & $-7 e$ & $-13 e$ \\
Slow transition [29] & $-14 e$ & $-13 e$ \\
Fast transition [26] & $-2 e$ & $-1 e$ \\
\hline \hline
\end{tabular}

The results for both pressures are summarised in Tab.I
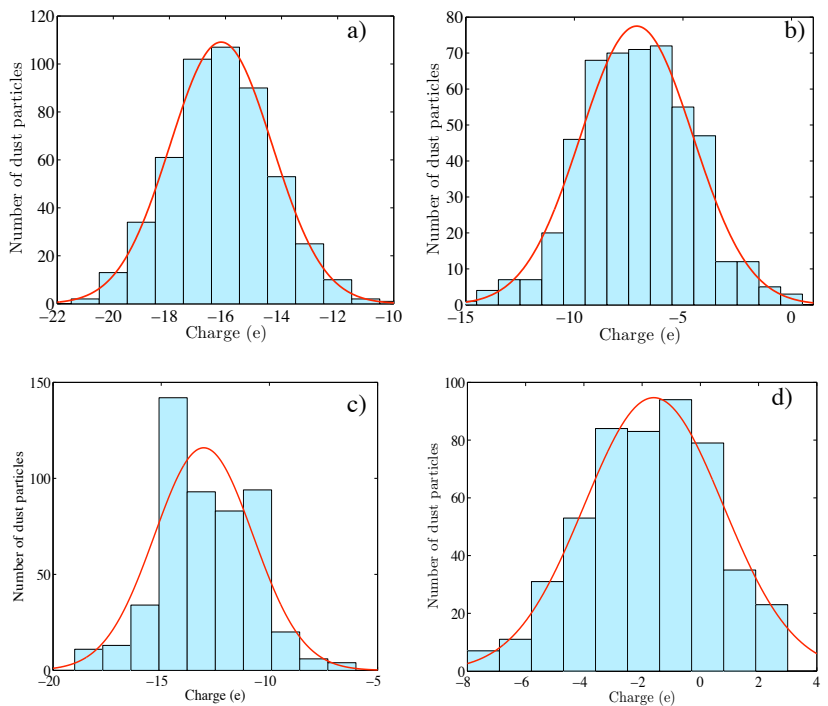

FIG. 6: (Color Online) Numerical results for 190 $\mathrm{nm}$ radius dust particles with argon pressure $P=1.2 \operatorname{mbar}(P=$ 0.9 Torr $)$ and $n_{d}=5 \cdot 10^{4} \mathrm{~cm}^{-3}$.a) Ambipolar diffusion until the end of the decay process. b) Abrupt transition from ambipolar to free diffusion when $P_{H}=0.5$. c) Using data from Gerber and Gerardo for transition from ambipolar to free diffusion [29]. d) Using data from Freiberg and Weaver for transition from ambipolar to free diffusion [26]

\section{DISCUSSION}

We now discuss the results and limits of our model. In general, we found that the transition from ambipolar to free diffusion plays a major role in the discharging process of the dust particle. It is instructive to see how the transition affects the residual dust charge, in particular, the width of dust charge distribution. The fact that electrons and ions began to diffuse independently at an early stage of afterglow changes the dust charge distribution function drastically.

As we can see from data in Fig.5(a,b,c) for the pressure of 0.4 mbar the distributions for ambipolar diffusion, abrupt and slow transitions do not differ significantly. So any difference in diffusion in the late afterglow (low ratio of $\left.\Lambda / \lambda_{D e}\right)$ has little effect on residual charge. In contrast, a slight difference at an early stage has a large influence on the final dust charge distribution and leads to the appearance of positively charge particles (Fig.5d). For the higher pressure $(1.2 \mathrm{mbar})$ this effect is not so pronounced, and the diffusion in the late afterglow plays a noteworthy role. In this case an abrupt transition directed due to the influence of the dust charge particle volume effect tends to decrease the residual dust particle charge (Fig.6b). It does not, however, give us the experimentally observed value of residual charge or the positive tail of the charge distribution. So the results obtained let us conclude that the four stage model can be used only for the rough estimation of residual charge. For a more accurate calculation one has to take into account the actual diffusive rates for the electrons and ions.

We now discuss the validity of the presented model. This model is valid only for low dust particle density. As the losses by diffusion are treated as in a dust-free plasma, the influence of dust particle has to be very small compared to the total process. Indeed if losses on dust particles are similar or bigger than those by diffusion, this last process must be significantly affected. It has thus been shown that the presence of a high density of dust particles significantly reduces the plasma loss time [37]. Moreover, if the total dust charge is not negligible compared that of ions and electrons, the diffusion process must also be modified as the dust particles will repel the electrons and attract the ions.

Consequently, the influence of dust particles can be treated independently from the other loss processes only if the total charge carried by the dust particles is small compared to the charge carried by electrons or ions during the decay process. Furthermore, losses on dust particle surfaces must not be the main loss process (i.e. $r_{\text {loss }}>>1$ ) to allow ion and electron diffusions to be treated in the same way as in dust-free plasma. This condition is definitely satisfied for a discharge plasma but could change during the afterglow so we have to calculate evolution of the ratio $n_{i_{\text {diff }}} / n_{i_{a b s}}$ in discharge afterglow where $n_{i_{\text {diff }}}$ is the ion density lost by diffusion and $n_{i_{a b s}}$ the ion density lost by absorption onto the dust particle surfaces.

It shows that this ratio stay more or less above 5 during the whole decay process regardless of the conditions of pressure or data used to take into account the transition from ambipolar to free diffusion (only results using Gerber and Gerardo data are presented in fig.7). It means that during the plasma decay, losses on dust particles are very small compared to the losses to the reactor walls and our model for plasma decay is valid to the end of the afterglow. Furthermore, looking at the ratio $\left|\bar{Q}_{d} n_{d} / e n_{i}\right|$ presented in Fig.8, it can be seen that, except at the very end of the afterglow (i.e. a few ms before the residual charges freeze), the total dust charge volume is much smaller than the total charge of ions. The charge onto the dust particles thus represents a negligible part of the 

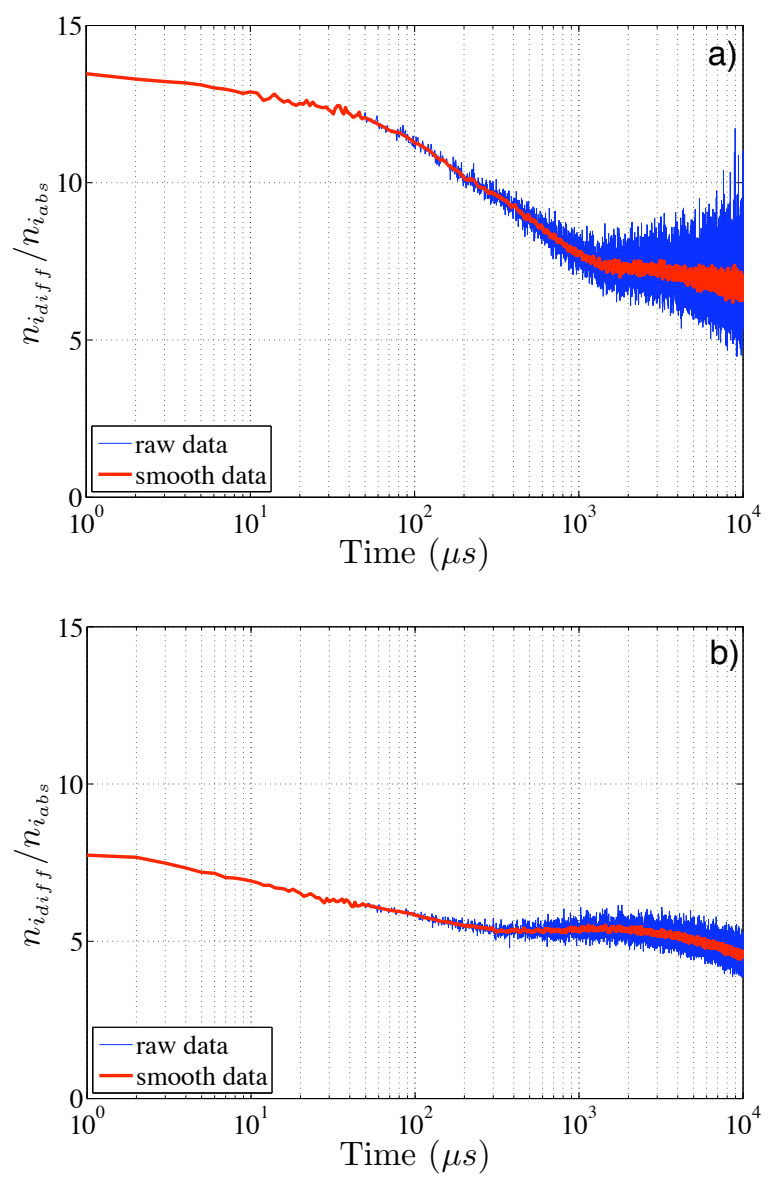

FIG. 7: (Color Online) Evolution of $n_{i_{d i f f}} / n_{i_{a b s}}$ using data from Gerber and Gerardo for transition from ambipolar to free diffusion [29]. a) $P=0.4$ mbar. b) $P=1.2 \mathrm{mbar}$

total charge during the major part of the decay process. The data presented in Figs. 7 and 8 confirmed that, in our case, the overall influence of dust particles on the plasma decay can be neglected and, consequently, the assumption of dust-free plasma diffusion can be considered as valid for the whole decay process.

It should be noted that the transition from ambipolar to free diffusion is not the only process that influence the dust residual charge. Indeed, it has been shown that in reactive plasmas such as $C_{2} H_{4} / A r$ plasma $[38,39]$ in which the dust density can be very high $\left.\left(n_{d} \simeq 4.5 \cdot 10^{6} \mathrm{~cm}^{-3}\right)[39]\right)$, the afterglow electron density shows an unexpected increase at the very beginning of the decay process. Berndt et al. attributed this increase to an electron release by the dust particles $[38,39]$. However, as a such increase has already been observed in pulsed helium discharges and attributed to a re-ionization due to metastable-metastable collisions $[40,41]$, and as the presence of high dust particle density in a argon dilution plasma enhances the metastable density [42], the increase of the electron density at the very beginning of the afterglow is still not clear. In all cases, these "extra electrons" must affect the charge on dust particles and consequently the residual charge distributions.

Moreover, it has been shown that the pressure in noble gases such as argon and neon significantly influences the diffusion coefficient [43]. Indeed, at low pressure the electron diffusion cooling process, i.e. the fast loss of energetic electrons to the walls of the reactor which are imperfectly compensated through elastic collisions of the electrons with gas atoms resulting in an electron temperature below that of the gas, causes a reduction of $D_{a} P$ and thus an enhancement of the ambipolar diffusion time $\tau_{D}=\Lambda^{2} / D_{a}$. As mentioned previously, the dust particle charges are related to ion and electron density. Diffusion cooling can also affect dust particle charge distribution evolution. Nevertheless, the present model (see Eq.6) does not take into account the phenomena of diffusion cooling and its importance has to be investigated in a future article.
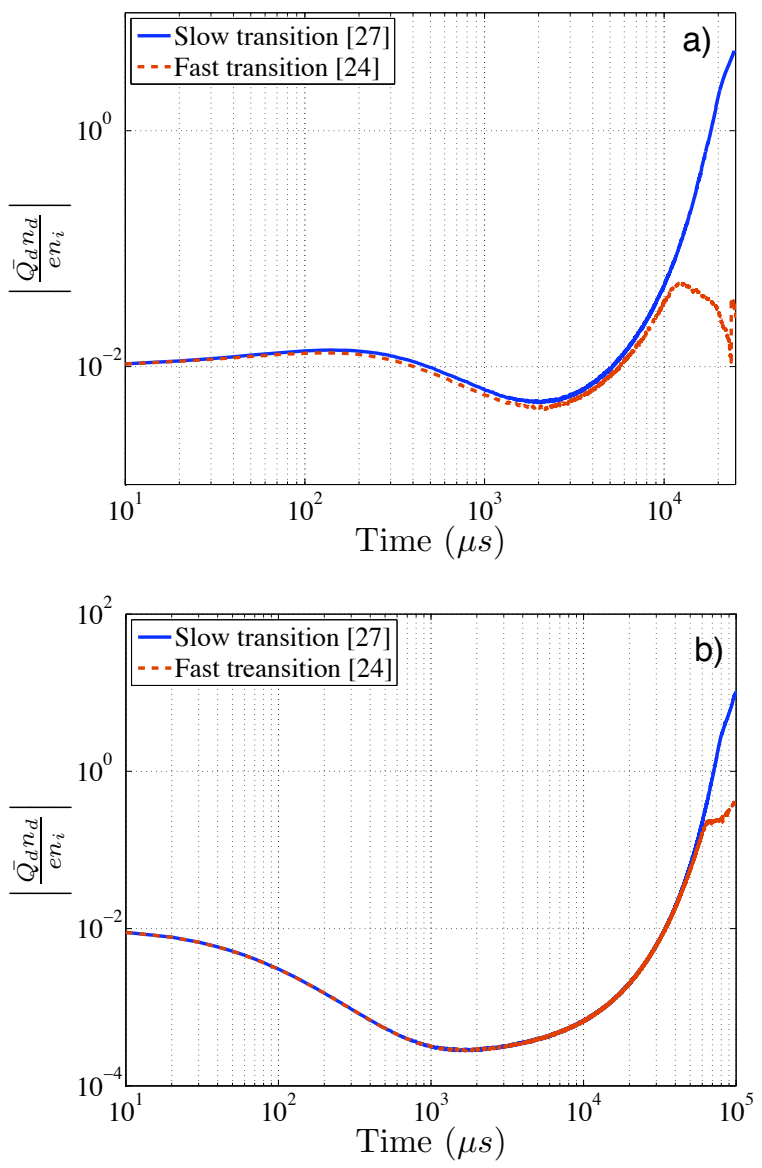

FIG. 8: (Color Online) Evolution of $\left|\overline{Q_{d}} n_{d} / e n_{i}\right|$. a) $P=$ 0.4 mbar. b) $P=1.2$ mbar 


\section{CONCLUSION}

To conclude, the decharging process of dust particles is strongly dependent on the transition from ambipolar to free diffusion as their charges depend on the ratio $n_{i} / n_{e}$ (see Eq.10 for equilibrium charge). In this article, it has been shown that the character of the transition influences the final residual dust charge distribution in a strong manner. The faster the transition occurs (the earlier electrons and ions start to diffuse separately), the smaller the mean dust particle residual charge and a positive charge tail can even be obtained. Consequently, it may be possible to use the dust particle charge distribution as a probe to study the transition from ambipolar to free diffusion in decaying plasma as this distribution is strongly dependent on the transition. Further investigations are being carried on about this possibility.

It has to be noted that the proposed model is valid only in a limited range of parameters as some important issues such as the influence of dust particle charge volume, possible metastable re-ionization, low pressure diffusion cooling, have been ignored. Consequently, further studies are needed for a fuller understanding of the decay process in dusty plasmas.

\section{Acknowledgments}

The authors would like to thank S. Dozias and B. Dumax for electronic support, J. Mathias for optical support, Y. Tessier, C. Cuthbert and J. Sisovic for experimental support. The authors would also like to thanks B. W. James for helpful discussions. The PKE-Nefedov chamber has been made available by the Max-Planck-Institute for Extraterrestrial Physics, Germany, under the funding of DLR/BMBF under grants No.50WM9852. This work was supported by CNES under contract $793 / 2000 / \mathrm{CNES} / 8344$. This paper is partially supported by the French-Australian integrated research program (FAST) under contract No.FR060169.
[1] A. P. Nefedov, G. E. Morfill, V. E. Fortov, H. M. Thomas, H. Rothermel, T. Hagl, A. V. Ivlev, M. Zuzic, B. A. Klumov, A. M. Lipaev, V. I. Molotkov, O. F. Petrov, Y. P. Gidzenko, S. K. Krikalev, W. Shepherd, A. I. Ivanov, M. Roth, H. Binnenbruck, J. A. Goree, Y. P. Semenov, New J. Phys. 5, 33 (2003).

[2] A. Bouchoule and L. Boufendi, Plasma Sources Sci. Technol. 2, 204 (1993).

[3] M. Cavarroc, M. C. Jouanny, K. Radouane, M. Mikikian, and L. Boufendi, J. Appl. Phys. 99, 064301 (2006).

[4] M. Mikikian, L. Boufendi, A. Bouchoule, H. M. Thomas, G. E. Morfill, A. P. Nefedov, V. E. Fortov, and the PKENefedov team, New J. Phys. 5, 19 (2003).

[5] M. Mikikian and L. Boufendi, Phys. Plasmas 11, 3733 (2004).

[6] M. Mikikian, M. Cavarroc, L. Couëdel, and L. Boufendi, Phys. Plasmas 13, 092103 (2006).

[7] D. Samsonov and J. Goree, J. Vac. Sci. Technol. A 17, 2835 (1999).

[8] A. A. Samarian and B. W. James, Phys. Lett. A 287, 125 (2001).

[9] M. Mikikian, L. Couëdel, M. Cavarroc, Y. Tessier, and L. Boufendi, New J. Phys. 9, 268 (2007).

[10] S. Vladimirov, K. Ostrikov, and A. Samarian, Physics and Applications of Complex Plasmas (Imperial Press, London, 2005).

[11] A. Bouchoule, Dusty Plasmas: Physics, Chemistry and Technological impacts in Plasma Processing (Wiley, New York, 1999).

[12] S. Matsusaka, M. Oki, and H. Masuda, Powder Technology 135-136, 150 (2003).

[13] K. Schonert, K. Eichas, and F. Niermoller, Powder Technology 86, 41 (1996).

[14] S. Anisimov, E. Mareev, N. Shikhova, A. Sorokin, and E. Dmitriev, Atmospheric Research 76, 16 (2005).

[15] H. Kersten, H. Deutsch, and G. M. W. Kroesen, Int. J. Mass Spectr. 233, 51-60 (2004).
[16] E. B. Tomme, D. A. Law, B. M. Annaratone, and J. E. Allen, Phys. Rev. Lett. 85, 2518 (2000).

[17] C. Zafiu, A. Melzer, and A. Piel, Phys. Rev. E 63, 066403 (2001).

[18] A. A. Samarian and S. V. Vladimirov, Phys. Rev. E 67, 066404 (2003)

[19] O. S. Vaulina, A. A. Samarian, B. James, O. F. Petrov, and V. E. Fortov, JETP 96, 1037 (2003).

[20] N. J. Prior, L. W. Mitchell, and A. A. Samarian, J. Phys. D: Appl. Phys. 36, 1249 (2003).

[21] S. Ratynskaia, S. Khrapak, A. Zobnin, M. H. Thoma, M. Kretschmer, A. Usachev, V. Yaroshenko, R. A. Quinn, G. E. Morfill, O. Petrov, V. Fortov, Phys. Rev. Lett. 93, 085001 (2004).

[22] C. Arnas, M. Mikikian, and F. Doveil, Phys. Rev. E 60, 7420 (1999).

[23] M. A. Childs and A. Gallagher, J. Appl. Phys. 87, 1086 (2000).

[24] A. Ivlev, M. Kretschmer, M. Zuzic, G. E. Morfill, H. Rothermel, H. Thomas, V. E. Fortov, V. I. Molotkov, A. P. Nefedov, A. M. Lipaev, O. F. Petrov, Yu. M. Baturin, A. I. Ivanov, J. Goree, Phys. Rev. Lett. 90, 055003 (2003).

[25] L. Couëdel, M. Mikikian, L. Boufendi, and A. A. Samarian, Phys. Rev. E 74, 026403 (2006).

[26] R. J. Freiberg and L. A. Weaver, Phys. Rev. 170, 336 (1968).

[27] M. A. Gusinow and R. A. Gerber, Phys. Rev. A 5, 1802 (1972).

[28] R. A. Gerber, M. A. Gusinow, and J. B. Gerardo, Phys. Rev. A 3, 1703 (1971).

[29] R. A. Gerber and J. B. Gerardo, Phys. Rev. A 7, 781 (1973).

[30] Y. P. Raizer, Gas Disharge Physics (Springer, Berlin, 1991).

[31] R. N. Varney, Phys. Rev. 88, 362 (1952).

[32] P. K. Shukla and A. A. Mamun, Introduction to dusty 
plasma physics (IOP Publishing, 2002).

[33] T. Matsoukas and M. Russell, J. Appl. Phys. 77, 4285 (1995).

[34] C. Cui and J. Goree, IEEE Trans. Plasma Sci. 22, 151 (1994).

[35] H. Rothermel, T. Hagl, G. E. Morfill, M. H. Thoma, and H. Thomas, Phys. Rev. Lett. 89, 175001 (2002).

[36] B. Kivel, Phys. Rev 116, 926 (1959).

[37] K. Dimoff and P. R. Smy, Phys. Lett. A 32, 13 (1970).

[38] J. Berndt, E. Kovacevic, V. Selenin, I. Stefanovic, and J. Winter, Plasma Sources Sci. Technol. 15, 18 (2006).
[39] I. Stefanovic, J. Berndt, D. Maric, V. Samara, M. Radmilovic-Radjenovic, Z. L. Petrovic, E. Kovacevic, and J. Winter, Phys. Rev. E 74, 026406 (2006).

[40] M. A. Biondi, Phys. Rev. 82, 453 (1951).

[41] L. J. Overzet and J. Kleber, Plasma Sources Sci. Technol. 7, 512 (1998).

[42] A. Bouchoule and L. Boufendi, Plasma Sources Sci. Technol. 3, 292 (1994).

[43] M. A. Biondi, Phys. Rev. 93, 1136 (1954). 